\title{
DTM-Pade Approximants for MHD Flow with Suction/Blowing
}

\author{
M. Thiagarajan ${ }^{\dagger}$, K. Senthilkumar \\ Department of Mathematics,PSG College of Arts and Science, Coimbatore-641014, India
}

†Corresponding Author Email: thiyagu26@rediffmail.com

(Received April 17, 2012; accepted October 17, 2012)

\begin{abstract}
In this paper, we study theoretically the magnetic effect of Blasius equation with suction/blowing. The similarity transformations are applied to reduce the governing partial differential equations to a set of nonlinear ordinary differential equations in dimensionless form. A mathematical technique, namely the Differential Transform Method (DTM), is used to solve the nonlinear differential equations under appropriate boundary conditions, in the form of series with easily computable terms. Then, Pade approximants are applied to the solutions to increase the convergence of the given series. The combined DTM-Pade procedure is implemented directly without requiring linearization, discretization or perturbation. Graphical results are presented to investigate influence of the Magnetic field on the velocity profiles.
\end{abstract}

Keywords: MHD, Porosity, Differential Transformation Method (DTM), Pade approximation.

\section{INTRODUCTION}

The study of magnetohydrodynamic incompressible viscous flow has many important engineering applications in devices such as power generator, the cooling of reactors, the design of heat exchangers and MHD accelerators. In hydromagnetics, the problem of Hiemenz flow was chosen by $\mathrm{Na}$ (1979) to illustrate the solution of a third-order boundary value problem using the finite difference method. Ariel (1994) has considered the stagnation-point flow of electricallyconducting fluids in the presence of large transverse magnetic field strengths.

There has been an interest in studying magnetohydrodynamic flow in porous and non-porous media due to the effect of magnetic fields on the performance of many systems using electrically conducting fluids. Raptis et al. (1982) have analyzed hydromagnetic free convection flow through a porous medium between two parallel plates. Takhar and Ram (1994) have studied MHD forced and free convection flow of water at $4^{\circ} \mathrm{C}$ through a porous medium in the presence of a uniform transverse magnetic field for the local similarity equations.

Because of the nonlinearities in the reduced differential equation, no analytical solution is available and the nonlinear equations usually are solved numerically subject to boundary conditions, one of which is prescribed at infinity. Although with the advancement of the symbolic computation software such as MATHEMATICA, MAPLE, MATLAB and so on, approximate analytic methods for nonlinear problems have been adopted by many researchers.

Wazwaz (2001) investigated an efficient analytical and numerical procedure for solving boundary value problems for higher- order integro-differential equations by using Adomian decomposition method. Wazwaz (2006) examined the modified decomposition method is applied for analytic treatment of nonlinear differential equations that appear on boundary layers in fluid mechanics. The modified method accelerates the rapid convergence of the series solution, dramatically reduces the size of work. The series solution is combined with the diagonal Pade approximants to handle the boundary condition at infinity.

Wazwaz (2007) analyzed a reliable treatment of two forms of the third order nonlinear Blasius equation which comes from boundary layer equations by solving variational iteration method. This study shows that the series solution is obtained without restrictions on the nonlinearity behavior. Kuo (2004) investigated thermal boundary-layer problems in a semi-infinite flat plate by the differential transformation method. The differential transformation method is used to determine some solutions of these velocity and thermal boundary-layer problems.

In recent years, the DTM has been successfully employed to solve many types of nonlinear problems such as the linear partial differential equations of fractional order Odibat and Momani (2008), multi-order fractional differential equations Erturk et al. (2008), the 
hyperchaotic Rossler system Al-Sawalha et al. (2009), the fourth-order boundary value problems Erturk and Momani (2007), the Volterra integral equation with separable kernels Odibat (2008), the differentialdifference equations Arikoglu and Ozkol (2008), the free vibration equations of beam on elastic soil Catal (2008), the integral and integro-differential equation systems Arikoglu and Ozkol (2008). All of these successful applications verified the validity, effectiveness and flexibility of the DTM.

This method constructs for differential equations an analytical solution in the form of a power series. Furthermore, power series are not useful for large values of $\eta$, say $\eta \rightarrow \infty$. It is now well known that Pade approximants Baker (1975), Baker and Graves-Morris (1981) have the advantage of manipulating the polynomial approximation into rational functions of polynomials. It is therefore essential to combination of the series solution, obtained by the DTM with the Pade approximant Peker et al. (2011), Rashidi and Erfani (2011) to provide an effective tool to handle boundary value problems at infinite domains.

In this present problem, the influence of magnetic effect of Blasius equation with suction/blowing is analyzed; the nonlinear partial differential equation is solved for the velocity distribution. The effect of magnetic parameter and suction/blowing parameter on dimensionless velocity is thoroughly investigated.

\section{Problem Statement MATHEMATICAL FORMULATION}

AND

Many different but related phenomena are stated and studied by the Blasius equation Arikoglu and Ozkol (2005), Fang et al. (2008), Fang and Lee (2005) that has a special importance for all boundary-layer equations in fluid mechanics. The Blasius equation can be described as the non-dimensional velocity distribution in the laminar boundary layer over a semi-infinite flat plate which is shown in Fig.1.

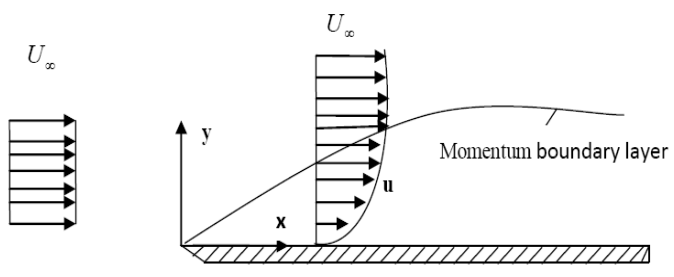

Fig. 1. Velocity layer on a flat plate

It is assumed that the free stream velocity, $U_{\infty}$, is also uniform and constant. Further, the incompressible viscous fluid is electrically conducting under the influence of an applied magnetic field normal to the stretching sheet. The induced magnetic field is neglected. The boundary-layer equations are as follows

$\frac{\partial u}{\partial x}+\frac{\partial v}{\partial y}=0$ $u \frac{\partial u}{\partial x}+v \frac{\partial u}{\partial y}=v \frac{\partial^{2} u}{\partial y^{2}}-\frac{\sigma B_{0}^{2}}{\rho} u$

Where $\mathrm{u}$ and $\mathrm{v}$ are the velocity components in $\mathrm{x}$ and $\mathrm{y}$ direction of the fluid, $v$ is the viscosity of the fluid, $\sigma$ is the electrical conductivity of the fluid in Eq. (2), the external electric field and polarization effects are negligible, $\rho$ is the density and $B_{0}$ is the externally imposed magnetic field in the y-direction.

The boundary conditions are

at $y=0: u=0: v=v_{w}$

at $y=0: u=0: v=v_{w}$

at $x=0: u=U_{\infty}$

where $v_{w}$ is the velocity across the stretching sheet when $v_{w}<0$, and it is blowing velocity when $v_{w}>0$.

A stream function, $\psi(x, y)$, is introduced such that

$u=\frac{\partial \psi}{\partial y} \quad$ and $\quad v=-\frac{\partial \psi}{\partial x}$

In addition to the physical considerations which require the introduction of this function, the mathematical significance of its use is that the equation of continuity, i.e. Eq. (1), is satisfied identically, and the momentum equation becomes:

$\frac{\partial \psi}{\partial y} \frac{\partial^{2} \psi}{\partial x \partial y}-\frac{\partial \psi}{\partial x} \frac{\partial^{2} \psi}{\partial y^{2}}=v \frac{\partial^{3} \psi}{\partial y^{3}}-\frac{\sigma B_{0}^{2}}{\rho} \frac{\partial \psi}{\partial y}$

Introducing a similarity variable Kuo (2004)

$f(\eta)=\frac{\psi}{\sqrt{U_{\infty} V x}}$

where

$\eta=y \sqrt{\frac{U_{\infty}}{v x}}$

Substituting Eqs. (8) and (9) with Eq. (6) into Eq. (7) gives

$f^{\prime \prime \prime}(\eta)+\frac{1}{2} f(\eta) f^{\prime \prime}(\eta)-M f^{\prime}(\eta)=0$

where $M=\frac{\sigma B_{0}^{2}}{\rho U_{\infty}}$ is the magnetic parameter.

The boundary conditions Wazwaz (2001), Wazwaz (2006) of $f(\eta)$ are given by

$f(0)=S, f^{\prime}(0)=1, f^{\prime}(\infty)=0$

where $\mathrm{S}$ is suction/blowing parameter $(\mathrm{S}<0$ is suction and $\mathrm{S}>0$ is blowing).

\section{DIFFERENTIAL TRANSFORMATION METHOD}


Consider a function $\mathrm{u}(\mathrm{x})$ which is analytic in a domain $\mathrm{T}$ and let $\mathrm{x}=\mathrm{x}_{0}$ represent any point in $\mathrm{T}$. The function $\mathrm{u}(\mathrm{x})$ is then represented by a power series whose center is located at $\mathrm{x}_{0}$. The differential transform of the function $\mathrm{u}(\mathrm{x})$ is given by

$$
U(k)=\frac{1}{k !}\left[\frac{d^{k} u(x)}{d x^{k}}\right]_{x=x_{0}}
$$

Where $u(x)$ is the original function and $U(k)$ is the transformed function. The inverse transformation is defined as follows

$$
u(x)=\sum_{k=0}^{\infty}\left(x-x_{0}\right)^{k} U(k)
$$

Combining Eq. (12) and Eq. (13), we get

$$
u(x)=\sum_{k=0}^{\infty} \frac{\left(x-x_{0}\right)^{k}}{k !}\left[\frac{d^{k} u(x)}{d x^{k}}\right]_{x=x_{0}}
$$

Inspection of Eq. (14) indicates that the concept of differential transform is derived from Taylor series expansion. However, this method does not evaluate the derivates symbolically. In actual applications, the function $\mathrm{u}(\mathrm{x})$ is expressed by a finite series and Eq. (13) can be rewritten as follows:

$u(x) \cong \sum_{k=0}^{m}\left(x-x_{0}\right)^{k} U(k)$

Which means that $u(x) \cong \sum_{k=m+1}^{\infty}\left(x-x_{0}\right)^{k} U(k)$ is negligibly small. Usually, the value of $\mathrm{m}$ is decided by convergence of the series coefficients.

\section{The Pade Approximants}

Suppose that we are given a power series $\sum_{i=0}^{\infty} a_{i} x^{i}$, representing a function $f(x)$, so that

$f(x)=\sum_{i=0}^{\infty} a_{i} x^{i}$

The Pade approximant is a rational fraction and the notation for such a Pade approximant is Baker (1975), Baker and graves-Morris (1981).

$[L, M]=\frac{P_{L}(x)}{Q_{M}(x)}$

Where $P_{L}(x)$ is a polynomial of degree at most $\mathrm{L}$ and $Q_{M}(x)$ is a polynomial of degree at most M. we have:

$$
\begin{aligned}
& f(x)=a_{0}+a_{1} x+a_{2} x^{2}+a_{3} x^{3}+\ldots \\
& P_{L}(x)=p_{0}+p_{1} x+p_{2} x^{2}+\ldots+p_{L} x^{L}
\end{aligned}
$$

$Q_{M}(x)=q_{0}+q_{1} x+q_{2} x^{2}+\ldots+q_{M} x^{M}$

notice that in Eq.(17) there are $\mathrm{L}+1$ numerator coefficients and M+1 denominator coefficients. Since we can clearly multiply the numerator and denominator by a constant and leave [L, M] unchanged, we impose the normalization condition

$Q_{M}(0)=1$

So there are $\mathrm{L}+1$ independent numerator coefficients and $\mathrm{M}$ independents denominator coefficients, making $\mathrm{L}+\mathrm{M}+1$ unknown coefficient in all. This number suggests that normally the $[\mathrm{L}, \mathrm{M}]$ ought to fit the power series Eq. (16) through the orders $1, x, x^{2}, \ldots, x^{L+M}$. Using the conclusion given in Baker (1975), Baker and graves-Morris (1981), we know that the [L, M] approximant is uniquely determined.

If the notation of formal power series,

$$
\sum_{i=0}^{\infty} a_{i} x^{i}=\frac{p_{0}+p_{1} x+p_{2} x^{2}+p_{3} x^{3}+\ldots+p_{L} x^{L}}{q_{0}+q_{1} x+q_{2} x^{2}+q_{3} x^{3}+\ldots+q_{M} x^{M}}+0\left(x^{L+M+1}\right)
$$

By cross-multiplying Eq. (22), we find that

$$
\begin{aligned}
& \left(a_{0}+a_{1} x+a_{2} x^{2}+a_{3} x^{3}+\ldots\right) \\
& \left(1+q_{1} x+q_{2} x^{2}+q_{3} x^{3}+\ldots+q_{M} x^{M}\right) \\
& =p_{0}+p_{1} x+p_{2} x^{2}+p_{3} x^{3}+\ldots+p_{L} x^{L}+0\left(x^{L+M+1}\right)
\end{aligned}
$$

From Eq. (23) one can obtained the set of equations

$\left\{\begin{array}{l}a_{0}=p_{0}, \\ a_{1}+a_{0} q_{1}=p_{1}, \\ a_{2}+a_{1} q_{1}+a_{0} q_{2}=p_{2}, \\ \vdots \\ a_{L}+a_{L-1} q_{1}+\ldots+a_{0} q_{L}=p_{L}\end{array}\right.$

$\left\{\begin{array}{l}a_{L-1}+a_{L} q_{1}+\ldots+a_{L-M+1} q_{M}=0 \\ a_{L-2}+a_{L+1} q_{1}+\ldots+a_{L-M+2} q_{M}=0 \\ \vdots \\ a_{L-M}+a_{L-M-1} q_{1}+\ldots+a_{L} q_{M}=0\end{array}\right.$

where $a_{n}=0$ for $\mathrm{n}<0$ and $q_{j}=0$ for $\mathrm{j}>$ M. If Eqs. (24) and (25) are nonsingular, then we can solve them directly

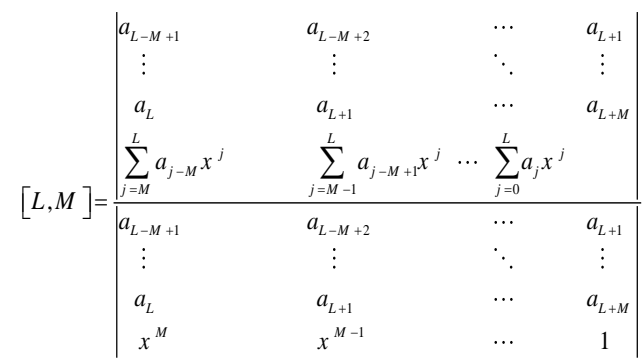

If the lower index on a sum exceeds the upper, the sum is replaced by zero. Alternate forms are 


$$
\begin{aligned}
& {[L, M]=\sum_{j=0}^{L-M} a_{j} x^{j}+x^{L-M+1} w_{L / M}^{T} W_{L / M}^{-1} w_{L / M}} \\
& =\sum_{j=0}^{L+n} a_{j} x^{j}+x^{L+n-1} w_{(L+M) / M}^{T} W_{L / M}^{-1} w_{(L+n) / M} . \\
& W_{L, M}=\left[\begin{array}{ccc}
a_{L-M+1}-x a_{L-M+2} & \cdots & a_{L}-x a_{L+1} \\
\vdots & \ddots & \vdots \\
a_{L}-x a_{L+1} & \cdots & a_{L+M+1}-x a_{L+M}
\end{array}\right], \\
& w_{L, M}=\left[\begin{array}{c}
a_{L-M+1} \\
a_{L-M+2} \\
\vdots \\
a_{L}
\end{array}\right]
\end{aligned}
$$

The construction of $[\mathrm{L}, \mathrm{M}]$ approximants involves only algebraic operations Baker (1975), Baker and GravesMorris (1981). Each choice of L, degree of the numerator and $\mathrm{M}$, degree of the denominator, leads to an approximant. The major difficulty in applying this technique is how to direct the choice in order to obtain the best approximant. This needs the use of a criterion for the choice depending on the shape of the solution. A criterion which has worked well here is the choice of $[\mathrm{L}, \mathrm{M}]$ approximants such that $\mathrm{L}=\mathrm{M}$. More importantly, the diagonal approximant is the most accurate approximant, therefore we will construct only the diagonal approximants in the following discussions.

\section{AnAlytical APPROXimations BY MEANS OF THE DTM-PADE}

The fundamental mathematical operations performed by DTM are listed in Table 1. taking the differential transform of Eq. (10), we obtain

$$
\begin{aligned}
& (k+1)(k+2)(k+3) F(k+3) \\
& +\frac{1}{2} \sum_{r=0}^{k}(k-r+2)(k-r+1) F(r) F(k-r+2) \\
& -M(k+1) F(k+1)=0
\end{aligned}
$$

Where $\mathrm{F}(\mathrm{k})$ is the differential transform of $f(\eta)$. The transform of the boundary conditions are:

$$
F(0)=0, F(1)=1, F(2)=\alpha
$$

Moreover, substituting Eq. (31) into Eq. (30) and by a recursive method we can calculate the values of $\mathrm{F}[\mathrm{k}]$, where $\alpha$ is a constant that is computed from the boundary condition.

For computing their values, the problem is solved with initial condition Eq. (31) and boundary conditions Eq. (11) are applied. The ideal method for enlarging the convergence radius of the truncated series solution is the Pade approximant i.e. converting the polynomial approximation into a ratio of two polynomials. Without using the Pade approximant, the analytical solution obtained by the DTM, cannot satisfy boundary conditions at infinity. It is therefore essential to combine the series solution, obtained by DTM with the Pade approximant to provide an effective tool for accommodating boundary value problems in infinite domains.

\section{RESULTS AND DISCUSSIONS}

An extensive range of computations has been performed with both numerical shooting method and DTM-Pade methods. The two-point boundary value problem for boundary layer convection from a flat plate is evidently governed two independent dimensionless parameters, namely $\mathrm{M}$ and $\mathrm{S}$. The far field boundary conditions must be applied in the computations at a finite value of the similarity variable $\eta$, here denoted by $\eta_{\max }$. In order to verify the accuracy of the present method, we have compared our results with those of numerical shooting method are presented in Table 2 and Table 3 .

Table 1 The operators for the one-dimensional differential transform method.

$$
\begin{aligned}
& w(x)=u(x) \pm v(x) \\
& w(x)=\lambda u(x) \\
& w(x)=x^{r} \\
& w(x)=\frac{d u(x)}{d x} \\
& w(x)=\frac{d^{r} u(x)}{d x^{r}} \\
& w(x)=u(x) v(x) \\
& w(x)=\frac{d u(x)}{d x} \frac{d v(x)}{d x} \\
& w(x)=u(x) \frac{d v(x)}{d x}
\end{aligned}
$$

$$
\begin{aligned}
& W(k)=U(k) \pm V(k) \\
& W(k)=\lambda U(k), \lambda \text { is a constant } \\
& W(k)=\delta(k-r), \text { where } \delta(k-r)=\left\{\begin{array}{l}
1, \text { if } k=r \\
0, \text { if } k \neq r
\end{array}\right. \\
& W(k)=(k+1) U(k+1) \\
& W(k)=(k+1)(k+2) \cdots(k+r) U(k+r) \\
& W(k)=\sum_{r=0}^{k} U(r) V(k-r) \\
& W(k)=\sum_{r=0}^{k}(r+1)(k-r+1) U(r+1) V(k-r+1) \\
& W(k)=\sum_{r=0}^{k}(k-r+1) U(r) V(k-r+1)
\end{aligned}
$$


M. Thiagarajan and K. Senthilkumar / JAFM, Vol. 6, No. 4, pp. 537-543, 2013.

Fig. 2 and Fig. 3 demonstrate the plot of dimensionless velocity field $f^{\prime}(\eta)$ for suction/blowing parameter $\mathrm{S}=$ $1,0,-1$ when $\mathrm{M}=0$ and $\mathrm{M}=1$ respectively.

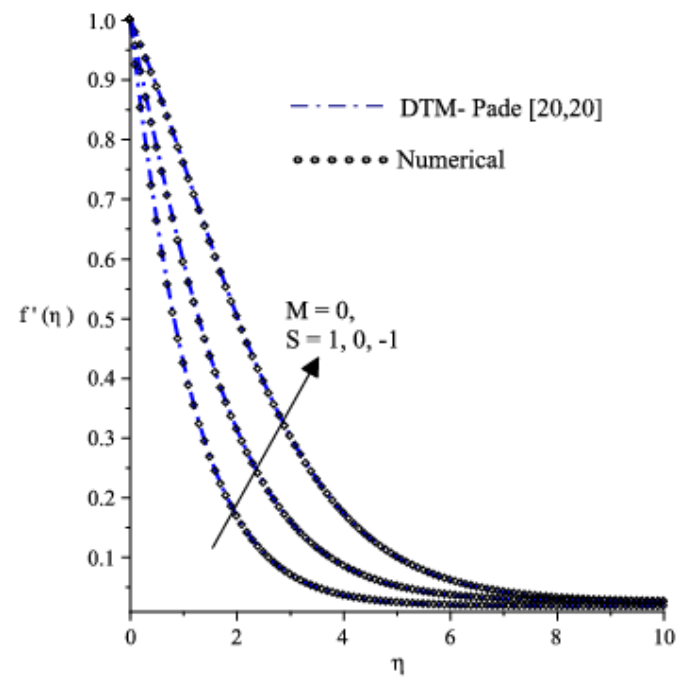

Fig. 2. Velocity profiles $f^{\prime}(\eta)$ for various values of porosity parameter $\mathrm{S}$ when $\mathrm{M}=0$.

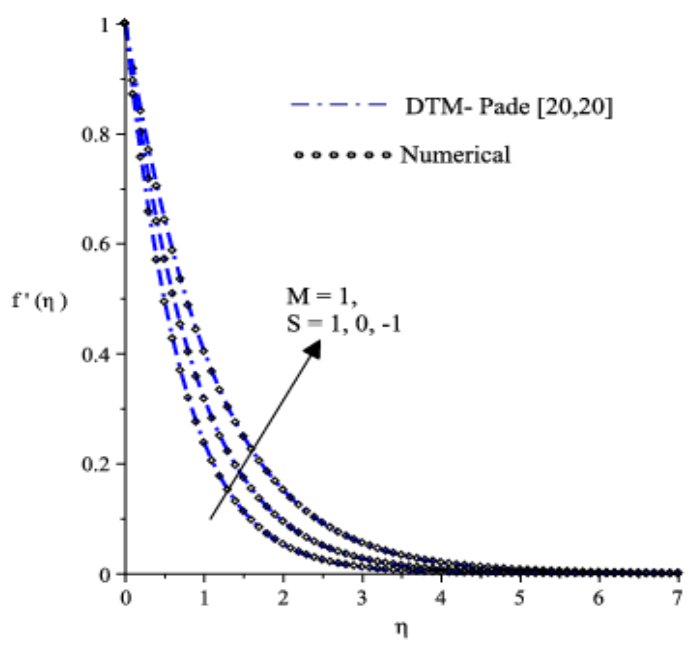

Fig. 3. Velocity profiles $f^{\prime}(\eta)$ for various values of porosity parameter $\mathrm{S}$ when $\mathrm{M}=1$.

Table 2 Various values of suction/blowing parameter $S$ obtained by the DTM-Pade and its numerical values for

\begin{tabular}{|c|c|c|}
\hline \multicolumn{3}{|c|}{$M=1$} \\
\hline S & DTM-Pade[20,20] & Numerical \\
\hline 0 & -0.8494260071 & -0.849425600 \\
\hline 1 & -1.082829541 & -1.082828844 \\
\hline
\end{tabular}

Fig. 4 displays the dimensionless velocity profile $f^{\prime}(\eta)$ for different values of Magnetic parameter M without suction/blowing parameter S. It is seen that the magnetic parameter M increases, velocity $f^{\prime}(\eta)$ decreases. Illustrating the fact that the effect of magnetic field is to decelerate the velocity.

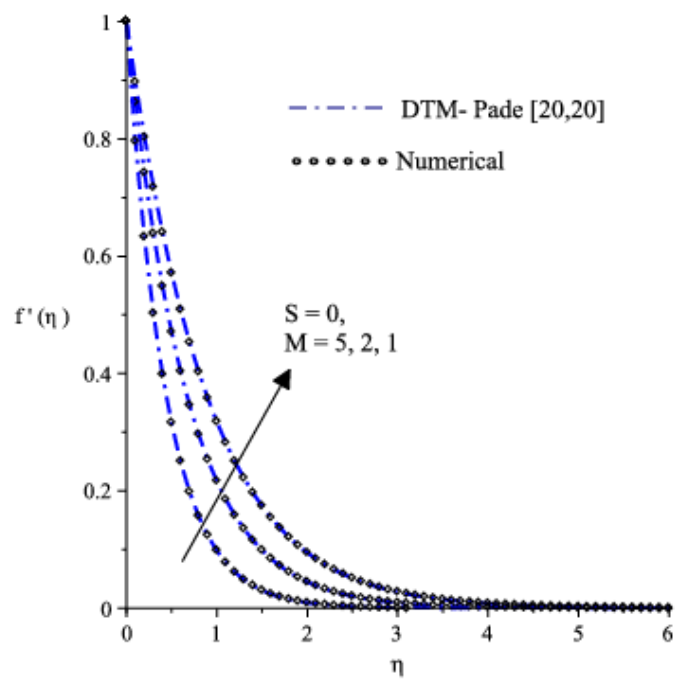

Fig. 4. Velocity profiles $f^{\prime}(\eta)$ for various values of Magnetic parameter $\mathrm{M}$ when $\mathrm{S}=0$.

The effect of magnetic parameter $\mathrm{M}$ with $\mathrm{S}>0$ over the dimensionless velocity $f^{\prime}(\eta)$ is represented graphically through Fig. 5. It is seen that the effect of magnetic field is to reduce the dimensionless velocity.

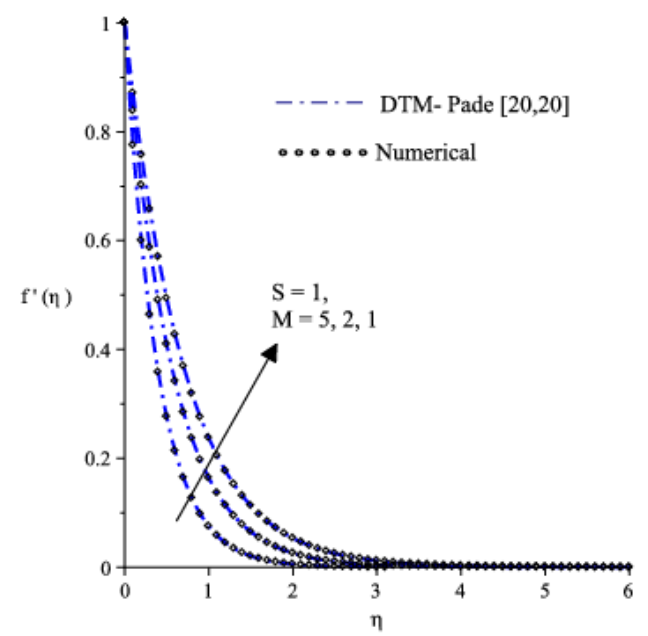

Fig. 5. Velocity profiles $f^{\prime}(\eta)$ for different values of Magnetic parameter $\mathrm{M}$ when $\mathrm{S}=1$.

Table 3 Various values of magnetic parameter $M$ obtained by the DTM-Pade and its numerical values for

\begin{tabular}{|c|c|c|}
\hline \multicolumn{3}{|c|}{ S $=0}$. \\
\hline M & DTM-Pade[20,20] & Numerical \\
\hline 1 & -1.082829541 & -1.082828844 \\
\hline 2 & -1.473041185 & -1.473039150 \\
\hline 5 & -2.273325296 & -2.273317103 \\
\hline
\end{tabular}

Dimensionless velocity $f^{\prime}(\eta)$ the different values of the magnetic parameter $M$ with $S<0$ is presented graphically in Fig. 6. It is noticed that for increasing values of magnetic parameter, the velocity 
$f^{\prime}(\eta)$ decreases which physically conveys the fact that the effect of magnetic field is to reduce the velocity

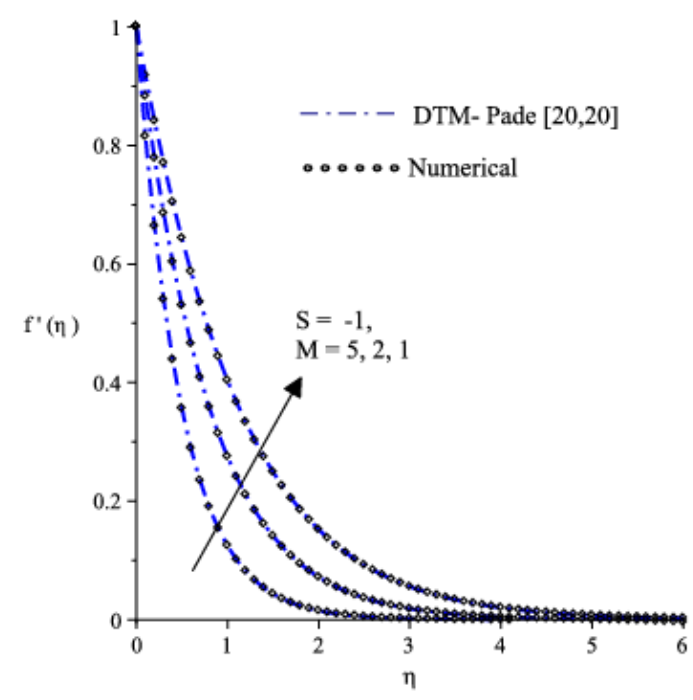

Fig. 6. Velocity profiles $f^{\prime}(\eta)$ for different values of Magnetic parameter $\mathrm{M}$ when $\mathrm{S}=-1$.

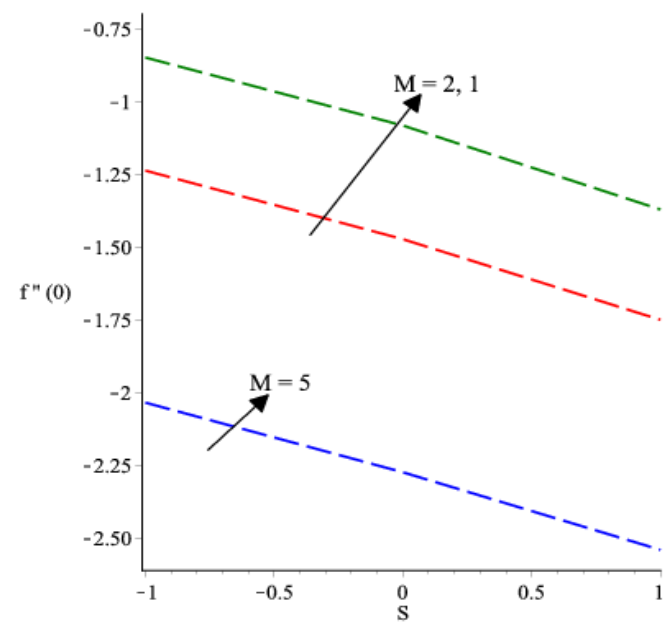

Fig. 7. Variation of $f$ "(0) with $\mathrm{S}$ for various values of Magnetic parameter M .

The effect of suction/blowing parameter $S$ on skin friction $f$ "(0) for different values of magnetic parameter $M$ is shown through Fig. 7. It is inferred that the effect of magnetic field and the effect of suction/blowing parameter have the similar effect over skin friction so as to reduce it.

Fig. 8 portrays the skin friction $f$ "(0) against magnetic parameter $\mathrm{M}$ for different suction/blowing parameter $\mathrm{S}$. It is observed that the effect of magnetic field is to decrease the skin friction. It is also seen that the skin friction decreases for $S>0$ whereas increases for $S<0$.

In the absence of magnetic field, Eq. (10) reduces to that of Peker et al (2011).

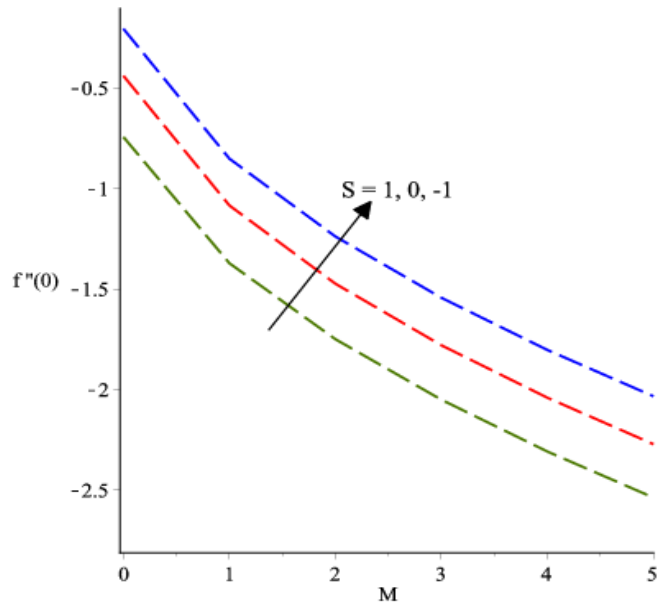

Fig. 8. Variation of $f$ "(0) with $\mathrm{M}$ for various values of porosity parameter $\mathrm{S}$.

\section{CONCLUSIONS}

The DTM-Pade is used to find analytical solutions of magnetohydrodynamics boundary-layer equations. The DTM combined with the Pade approximants are also shown to be a promising tool to solve two point boundary-value problems consisting of systems of nonlinear differential equations. This method has been applied directly without requiring linearization, discretization, or perturbation. The obtained results demonstrate the reliability of the algorithm and give it a wider applicability to nonlinear differential equations.

In the present problem, the influence of magnetic effect of Blasius equation is analyzed, the nonlinear partial differential equation is solved for the velocity distribution. Dimensionless velocity is reduced due to the influence of Magnetic field.

\section{REFERENCES}

Al-Sawalha, M.M. and M.S.M. Noorani (2009). Application of the differential transformation method for the solution of the hyperchaotic Rossler system. Communications in Nonlinear Science and Numerical Simulation, 14, 1509-1514.

Ariel, P.D. (1994). Hiemenz flow in hydromagnetics. Acta Mechanica, 103, 31-43.

Arikoglu, A. and I. Ozkol (2005). Inner-outer matching solution of Blasius equation by DTM, Aircraft Engineering and Aerospace Technology: An International Journal, 77, 298-301.

Arikoglu, A, and I., Ozkol, (2006). Solution of differential-difference equations by using differential transform method. Applied Mathematics and Computation, 181, 153-162. 
M. Thiagarajan and K. Senthilkumar / JAFM, Vol. 6, No. 4, pp. 537-543, 2013.

Arikoglu, A. and I. Ozkol (2008). Solutions of integral and integro-differential equation systems by using differential transform method. Computers \& Mathematics with Applications. 56, 2411-2417.

Baker, G.A. (1975). Essential of Pade approximants. London, Academic Press.

Baker, G.A. and P. Graves-Morris (1981). Encyclopedia of mathematics and its application, Parts I and II: Pade approximants. Addison-Wesley publishing company, New York.

Catal, S. (2008). Solution of free vibration equations of beam on elastic soil by using differential transform method. Applied Mathematical Modelling, 32, 1744-1757.

Erturk, V.S. and S. Momani (2007). Comparing numerical methods for solving fourth-order boundary value problems. Applied Mathematics and Computation, 188, 1963-1968.

Erturk, V.S., S. Momani and Z. Odibat (2008). Application of generalized differential transform method to multi-order fractional differential equations. Communications in Nonlinear Science and Numerical Simulation, 13, 1642-1654.

Fang, T. and C.F. Lee (2005). A moving-wall boundary layer flow of a slightly rarefied gas free stream over a moving flat plate, Applied Mathematical Letters, $18,487-495$.

Fang, T., W. Liang and C.F. Lee (2008). A new solution branch for the Blasius equation-A shrinking sheet problem, Computer and mathematics with applications, 56, 3088-3095.

Kuo, B.L. (2004). Thermal boundary-layer problems in a semi-infinite flat plate by the differential transformation method, Applied Mathematics and Computation, 150, 303-320.

Na, T.Y. (1979). Computational methods in engineering boundary value problem. Academic Press, New York.
Odibat, Z.M. (2008). Differential transform method for solving Volterra integral equation with separable kernels. Mathematical and Computer Modelling, 48, 1144-1149.

Odibat, Z. and S. Momani (2008). A generalized differential transform method for linear partial differential equations of fractional order. Applied Mathematics Letters, 21, 194-199.

Peker, H.A., O. Karaoglu and G. Oturanc (2011). The differential transformation method and Pade approximant for a form of classical Blasius equation. Mathematics and Computer Applications, 16(2), 507-513.

Raptis, A., C. Massalas and G. Tzivanidis (1982). Hydromagnetic free convection flow through a porous medium between two parallel plates. Physics Letters A., 90, 288-289.

Rashidi, M.M. and E. Erfani (2011). A new Analytical study of MHD stagnation-point flow in porous media with heat transfer, Computer and Fluids, 40, 172-178.

Takhar, H.S. and R.C. Ram (1994). Magnetohydrodynamic free convection flow of water at $4^{\circ} \mathrm{C}$ through a porous medium. International Communications in Heat and Mass Transfer. 21, 371-376.

Wazwaz, A. (2001). A reliable algorithm for solving boundary value problems for higher order integrodifferential equations, Applied Mathematics and Computation, 118, 327-342.

Wazwaz, A. (2006). The modified decomposition method and Pade approximation for a boundary layer equation in unbounded domain, Applied Mathematics and Computation, 177, 737-744.

Wazwaz. A. (2007). The variational iterational method for solving two forms of Blasius equation on a halfinfinite domain, Applied Mathematics and Computation, 188, 485-491. 